\title{
Pendulous Breast Radiotherapy in Prone Position
}

\section{Prone Pozisyonda Pandüler Meme Radyoterapisi}

\author{
Yasemin Güzle Adaş, Muzaffer Bedri Altundağ, Tamer Calikoglu, Serab Uyar, Ali Rıza Üçer, Gülçin \\ Ertaş, Atila Demirkasımoğlu, Sema Durmuş Düzgün, Hayati Abanuz, Erdal Demir, Kenan Özbağı
}

\author{
SBÜ Dr. Abdurrahman Yurtaslan Ankara Onkoloji EAH,Radyasyon Onkolojisi Kliniği,Ankara
}

\begin{abstract}
ÖZET
GİRIŞ ve AMAÇ: Meme koruyucu cerrahi uygulanmış meme kanseri tanılı hastalarda adjuvan radyoterapi standart tedavidir. Radyoterapi sonrası kozmetik sonuçları en çok etkileyen faktörlerden birisi de meme boyutudur. $\mathrm{Bu}$ çalışmanın amacı pandüler meme nedeni ile prone pozisyonda radyoterapi uyguladığımız hastalarda kozmezis ve dozimetrik dağılımının değerlendirilmesidir.YÖNTEM ve GEREÇLER: Temmuz 2015-Ağustos 2017 tarihleri arasında meme koruyucu cerrahi uygulanmış 17 hastaya prone pozisyonda radyoterapi uyguland. Hastalara prone meme yatağı ile simülasyon yapıldı ve konvansiyonel fraksinasyonla tüm memeye $50 \mathrm{~Gy}$; tümör yatağına ortanca 14 Gy (10-16 Gy) radyoterapi uygulandı. Tedavi planlama sisteminden hedef hacim ve kritik organların dozimetrik değerlendirmeleri yapıldı. RTOG tokisisite kriterleri kullanılarak akut ve geç yan etkiler değerlendirildi.

BULGULAR: Meme koruyucu cerrahi uygulanan büyük ve sarkık (panduler) memeli 17 hasta değerlendirildi. Ortalama yaş 59 (38-87); ortalama tümör boyutu $2.2 \mathrm{~cm}(0,7-5.5 \mathrm{~cm})$ idi. Tüm memeye $50 \mathrm{~Gy}$, tümör yatağına median 14 Gy (10-16 Gy) boost uyguland1. PTV meme minimum, maksimum, ortalama dozlar1; 3341cGy, 5544 cGy, 5161 cGy;.akciğer ortalama doz ve V5, V20, V30 ortalama yüzdeleri: 414 cGy, \%10.5,\% 5, \%3.6;kalp ortalama doz ve $\mathrm{V} 5, \mathrm{~V} 10, \mathrm{~V} 30$ ortalama yüzdeleri; $256 \mathrm{cGy}, \% 7.7, \% 3.3, \% 1.2$; karşı meme maksimum ve ortalama doz; 1128 cGy ve 44 cGy idi.7 hastada grade 2,1 hastada grade 3 radyodermit gelişti. Ortalama 14.5 aylık (1-24 ay) takipte nüks veya kötü kozmetik sonuç gözlenmedi.

TARTIŞMA ve SONUÇ: Panduler memelerin supine pozisyonda alınması ile daha çok doz inhomojenitesi görülmekte ve bunun sonucu olarak kozmezis bozulmaktadır. Ayrıca büyük alanların ışıllanması ile akciğer ve kalp toksisitesi artmaktadır. Panduler meme ş̧ınlamalarında prone pozisyon ile iyi doz homojenitesi ve kozmezis sağlanabilmektedir.
\end{abstract}

Anahtar Kelimeler: Meme kanseri, pandüler meme, prone pozisyon

\begin{abstract}
INTRODUCTION: Adjuvant radiotherapy is the standard treatment for breast cancer patients who underwent breast conserving surgery. One of the most important factors affecting cosmetic results after radiotherapy is breast size. The aim of this study was to evaluate the cosmesis and dosimetric distribution of patients undergoing radiotherapy in the prone position due to pandular breast.

METHODS: Between July 2015 and August 2017, 17 patients who had breast conserving surgery and radiotherapy were evaluated. Patients were simulated in prone position. Fifty Gy to the breast; median 14 Gy (10$16 \mathrm{~Gy}$ ) boost to the tumor bed were applied. Dosimetric results were evaluated from treatment planning system. Acute and late side effects were evaluated by using RTOG toxicity criteria.

RESULTS: Seventeen patients with pendulous breasts who underwent breast conserving surgery and radiotherapy were evaluated. The mean age was $59(38-87)$; mean tumor size was $2.2 \mathrm{~cm}(0.7-5.5 \mathrm{~cm})$. Median $50 \mathrm{~Gy}(48-50$ Gy) radiotherapy was applied to the whole breast with a median $14 \mathrm{~Gy}(10-16 \mathrm{~Gy})$ boost to the tumor bed. PTV minimum, maximum and mean doses were $3341 \mathrm{cGy}, 5544 \mathrm{cGy}, 5161 \mathrm{cGy}$; mean dose of lung and V5, V20, V30 values were $414 \mathrm{cGy}, 10.5 \%, 5 \%, 3.6 \%$; hear tmean dose and V5, V10, V30 values were; $256 \mathrm{cGy}, 7.7 \%, 3.3 \%$, $1.2 \%$; maximum and mean doses of the contrlateral breast were $1128 \mathrm{cGy}$ and $44 \mathrm{cGy}$. In 7 patients grade 2 radiodermitis; in 1 patient grade 3 radiodermitis was observed. There was no recurrence or poor cosmetic outcome during the14.5 months follow-up (1-24 months).
\end{abstract}


DISCUSSION AND CONCLUSION: More dose inhomogenity is seen in the supine position radiotherapy of pendulous breasts and as a result bad cosmetic results may be observed. Also lung and heart toxicity increases with the irradiation of large areas. Good dose homogenities and cosmetic results may be achieved by prone position.

Keywords: Breast cancer, pendulous breast, prone position

\section{GİRIS}

Meme kanseri kadınlarda en sık görülen ve ölüme neden olan kanserler arasında akciğer kanserinden sonra ikinci sirada yer alan kanserdir (1). Erken evre (evre I-II) meme kanserli olgular günümüzde genellikle meme koruyucu cerrahi ile tedavi edilmektedir. Meme koruyucu cerrahi uygulanmış olan hastalarda cerrahi sonrasi radyoterapi standart olarak önerilmektedir (2-5). Meme koruyucu cerrahi ve radyoterapi ile yapılan tedavinin amac1 düşük lokal nüks ile beraber iyi bir kozmetik sonuç elde etmektir.

Daha önce yapılan çalışmalarda meme koruyucu cerrahi sonrasi radyoterapi uygulanmış panduler memeye sahip hastalarda hem doz homojenitesinin hem de kozmetik sonuçların küçük memeye sahip hastalara göre daha kötü olduğu sonucuna varılmıştır. Özellikle cildin cilde temas ettiği meme sulkusunda cildinbolus etkisi yaratması sonucu bu bölgede doz artışları olduğu ve bunun da cilt yan etkilerinde artışa neden olduğu gözlenmiştir. Panduler memeye sahip hastalarda prone pozisyonda radyoterapi uygulamasının kozmetik sonuçlarının mükemmel olduğu ve 3 ve 5 y1llık lokal kontrol oranlarının da standart radyoterapi tekniklerine benzer olduğu belirtilmiştir (6-8).

$\mathrm{Bu}$ çalışmada meme koruyucu cerrahi sonrası prone pozisyonda radyoterapi uygulanmış olan hastalarda kozmetik sonuçların ve radyoterapi doz dağılımının değerlendirilmesi amaçlanmıştır.

\section{GEREÇ VE YÖNTEM}

Temmuz 2015-Kasım 2017 tarihleri arasında prone pozisyonda tedaviye alınmış 17 olgu retrospektif olarak değerlendirilmiştir. Çalışmaya erken evre meme kanseri tanısı ile meme koruyucu cerrahi sonrası radyoterapi (RT) uygulanmış olgular dahil edilmiştir.

RT öncesi tüm olguların fizik muayeneleri yapılmış, rutin biyokimya ve hemogram tetkikleri istenmiş, olgular patoloji raporları ve cerrahi öncesi istenmiş olan evreleme tetkikleri ile değerlendirilmiştir.
RT planlama için hastalara prone meme yatağ 1 (Q2 Fix Access Prone G2 Breast Device) ile pozisyonlama ve sabitleme yapılmış; 4D - BT simülatör (Philips Brillance CT Big Bore Oncology) ile 3 milimetrelik kesitlerle planlama tomografileri çekilmiştir. BT çekimi sonrası konturlama bilgisayarına gönderilen görüntüler üzerinden her hastaya hedef hacimler (tüm meme ve tümör boost dozu gereken olgularda tümör yatağı) klinik hedef alan olarak belirlenmiştir. Meme hedef hacmi (PTV) belirlenirken planlama BT'de görülen meme dokusu esas alınmıştır. Meme PTV'sine pektoral kaslar ve serratus anterior dahil edilmemiş; PTV sınırları lateralde mid-aksiller çizgiyi, medialde sternal-kostal bileşkeyi geçmeyecek şekilde belirlenmiştir. PTV ciltten $5 \mathrm{~mm}$ içeri çekilmiştir. Tümör yatağı cerrahi klipslerle belirlenmiş; cerrahi klips bulunmayan olgularda tümör yatağı belirlenmesi için cerrahi öncesi ultrasonografi ve mamografi görüntüleri ile birlikte; RT planlaması için çekilen planlama tomografilerindeki seroma görüntüleri birlikte değerlendirilmiştir. Tümör yatağı belirlendikten sonra her yönden $1.5 \mathrm{~cm}$ 'lik marjin verilerek tümör yatağ1 PTV'si oluşturulmuştur. Tüm hastalarda kritik organlar da (karşı meme, akciğerler, kalp) her BT kesitinde ayrı ayrı çizilmiştir.

Planlama Eclipse planlama sistemi ile field in field (FIF) tekniği kullanılarak yapılmış ve planlama için tanjansiyel alanlar kullanılmıştır. RT Varian Rapid Arc cihazı ile günlük 2 Gray (Gy) fraksiyon dozunda 25 fraksiyonda tüm memeye 50 Gy ; sonrasinda tümör yatağına median $14 \mathrm{~Gy}$ ( $10-16 \mathrm{~Gy}$ ) boost dozu olacak şekilde uygulanmıştır. Duktal karsinoma in situ tanis1 olan 2 olguya boost dozu uygulanmamıştır. 8 hastaya prone pozisyonda; 7 hastaya supin pozisyonda boost dozu uygulanmıştır.

Hastalar yan etkiler ve kozmetik sonuçlar açısından rutin kontrollerinde değerlendirilmiştir (RT süresince her hafta, RT'den 2 ay sonra, sonrasinda ise 2 yil boyunca 3 ayda bir). RT'ye bağlı cilt reaksiyonları RTOG (Radiation Therapy Oncology Group) toksisite değerlendirme kriterlerine göre 
skorlanmıştır. RTOG'nin değerlendirmelerine göre soluk, mat eritem, hafif gerginlik kaşıntı grade1; Hassasiyet, parlak eritem, kuru deskuamasyon, ağrı, kaşıntı ve gergin cilt grade 2; 1slak deskuamasyon, soyulma, ağrılı ödem, yeşi1/sarı eksüdagrade 3; ülserasyon, nekroz grade 4 olarak skorlanır (9). Dozimetrik değerlendirmeler ise planlama bilgisayarı üzerindeki veriler değerlendirilerek elde edilmiştir.Dozimetrik olarak hedef hacim minimum, maksimum , ortalama dozları ; akciğer ortalama dozu ve 5 Gy , 20 Gy ve 30 Gydoz alan hacim yüzdeleri ( V5, V10,V30); kalp ortalama ve V5,V10,V30 değerleri; karş1 meme maksimum ve ortalama dozları değerlendirilmiştir.

\section{BULGULAR}

Çalışmaya dahil edilen 17 hastanın 11'inde sağ meme; 6'sında sol meme kanseri tanıs1 mevcuttu. Ortalama yaş 59 (38-87), ortalama tümör boyutu $2.2 \mathrm{~cm}(0.7-5.5 \mathrm{~cm})$ olarak saptandı. Hastaların tamamına meme koruyucu cerrahi uygulanmıştı. 2 duktal karsinoma in situ (TisN0M0); 15 invaziv meme karsinom (T1-T2N0M0) tan1l hasta değerlendirildi. Hastalar hormon reseptör verilerine göre değerlendirildiğinde; 12 hastada ER ve PR pozitif, 2 hastada negatif bulunmuş; 3 hastanın ER ve PR verilerine ulaşılamamıştır. Her 2 verilerine göre değerlendirildiğinde 10 hastada Her 2 negatif, 4 hastada pozitif bulunmuş; 3 hastanın ise verilerine ulaşılamamıştır. Yedi hasta kemoterapi, 12 hasta hormonoterapi aldı. Panduler meme nedeni ile hastalara prone pozisyonda RT uygulanmasına karar verildi Tüm memeye 50 Gy, tümör yatağına median 14 Gy (10-16 Gy) boost uygulandi. DCIS tanıl1 2 hastaya boost uygulanmadi. 8 hastaya prone pozisyonda (7 foton, 1 elektron), 7 hastaya supin pozisyonda (5 elektron, 1 foton, 1 foton-elektron) boost uygulandi. Ortalama PTV meme hacmi $2094 \mathrm{cc}$ $(1467-3316$ cc) bulundu. PTV meme minimum, maksimum, mean ve D95 ortalama dozlar1; 3341cGy, 5544 cGy, 5161 cGy, 4785 cGy idi. Akciğer ortalama mean doz ve V5, V20, V30 ortalama yüzdeleri: 414 cGy, $\% 10.5, \%$ 5, \%3.6 idi. Kalp ortalama mean doz ve V5,V10,V30 ortalama yüzdeleri; 256cGy, \%7.7, \%3.3, \%1.2 idi. Karşı meme maksimum ve mean doz ortalamas1; 1128 cGy ve 44 cGy idi.
Hastaların tümünde RT'ye bağlı cilt değişiklikleri gözlendi, 9 hastada grade 1, 7 hastada grade 2, 1 hastada grade 3 akut radyodermit gelişti. Ortalama 14.5 aylık (1-24 ay) takipte nüks veya kötü kozmetik sonuç gözlenmedi.

\section{TARTIŞMA}

Meme koruyucu cerrahi ve adjuvan RT meme kanseri tanısı almış olan birçok kadın hastada mastektomiye eşdeğer sağkalım ve lokal kontrol sağlamaktadır. Ancak panduler memeye sahip kadınlarda cilt katlantılarında , özellikle meme sulkusunda oluşan yüksek dozdan dolayı RT ile ciddi akut cilt reaksiyonları ve sonrasinda fibrozis gözlenebilmektedir. Supin pozisyonda RT uygulanmış hastaların sonuçlarını değerlendiren çalışmalarda meme boyutunun büyümesi ile kozmetik sonucun kötüleştiği gözlenmiştir. Clarke ve arkadaşlarının tüm meme RT uygulanmış 78 hastayı değerlendirdikleri çalışmada A kap boyutundaki memelerde \%100 iyi kozmetik sonuç elde edilirken, D kap boyutundaki memelerde bu oranın \%50'ye düştüğü gözlenmiştir (10). Ray ve arkadaşlarının çalışmasında ise benzer şekilde A ve B kap boyutunda \% 92 mükemmel kozmetik sonuç elde edilirken, C kap ve üzerinde bu oran \%64'e gerilemiştir (11). Bu nedenle panduler memeye sahip olgularda RT'de prone pozisyonun kullanılması gündeme gelmiştir.

Bergom ve arkadaşlarının yaptığı çalışmada 1998-2006 yıllarında prone pozisyonda meme RT'si uygulanmış 110 olgunun kozmetik sonuçları değerlendirilmiş, en kötü gözlenen akut yan etki grade 3 olarak raporlanmış ve hastaların \%5'inde gözlenmiştir. Ortalama 40 aylık takip sonucunda hastaların \%89 'unda mükemmel kozmetik sonuç elde edilmiştir (12). Bizim çalışmamızda sadece 1 hastada grade 3 akut cilt yan etkisi gözlenmiş ve takiplerde hastaların \%100'ünde mükemmel kozmetik sonuç elde edilmiştir. $\mathrm{Bu}$ farklılık hem çalışmalardaki hasta sayılarının hem de hasta özelliklerinin (vücut kitle endeksi, kemoterapihormonoterapi kullanımı, ırk, cilt rengi gibi) farklılığından kaynaklanmış olabilir.

Chen ve arkadaşlarının 2013 yılında yaptı̆̆ 1 çalışmada prone pozisyonda tedaviye alınmış erken evre meme kanseri tanılı 21 hasta hem dozimetrik açıdan hem kozmetik sonuçlar açısından değerlendirilmiştir. Ortalama 26.8 aylık takipte hastaların $\% 88$ 'inde 
grade 1 dermatit gözlenmiş, grade 2 ve üzeri cilt yan etkisi gözlememiştir. Supin pozisyonla karşılaştırmalı değerlendirmede panduler memeye sahip bu olgularda prone pozisyon ile daha homojen doz dağılımı sağlandığı rapor edilmiştir (13).

Goodman ve arkadaşları erken evre meme kanseri nedeni ile RT uygulanmış panduler memeye sahip 20 hastada prone pozisyonda konvansiyonel tanjansiyel alan planları ile yoğunluk ayarlı radyoterapi (YART) plan sonuçlarını karşılaştırmışlardır. Konvansiyonel planda doz heterojenitesi daha yüksek bulunmuştur. Konvansiyonel planda maksimum dozlar \%114 olarak hesaplanırken YART planlarında bu değer \%107 olarak bulunmuştur. Yazarlar prone pozisyonda YART planlaması ile daha homojen doz dağılımı elde edildiğini bunun da akut ve kronik cilt yan etkilerinde azalma sağlayabileceğini belirtmişlerdir (14).

$\mathrm{Bu}$ çalışmadaki sonuçlarımız literatürdeki sonuçlar ile benzerdir. Sadece 1 hastada grade 3 akut yan etki gözlenmiş ve takiplerinde hastaların tamamında iyi kozmetik sonuç elde edilmiştir. Dozimetrik

\section{REFERANSLAR}

1. Siegel RL, Miller KD,Jemal A. Cancer Statistics.2017.CA Cancer J Clin 2017;67(1):7-30

2. Fisher B, Anderson S, Bryant J et al. Twentyyearfollow-up of a randomized trial comparing total mastectomy, lumpectomy, and lumpectomy plus irradiation for the treatment of invasive breast cancer. N Engl J med 2002;347:1233-41

3. Early Breast Cancer Trialists' Collobrative Group. Effects of radiotherapy and surgery in early breast cancer: an overview of the randomized trials .N Engl J med 1995;333:1444-55

4. Dongen JA, Voogd AC, Fentiman IS et al. Long term results of a randomized trial comparing breastconserving therapy with mastectomy: European Organization for research and treatment of cancer 10801 trial. J NatlCancerInst 2000;19:1143-50

5. Veronesi U, Cascinelli N, Mariani L et al. Twentyyear follow-up of a randomized study comparing breast - conserving surgery with radical mastectomy for early breast cancer. $\mathrm{N}$ Engl. J Med 2002;347:1227-32

6. Grann A, McCormick B, Chabner ES et al.Prone breast radiotherapy in early-stage breast cancer: a preliminary analysis.Int $\mathbf{J}$ Radiat OncolBiolPhys. $2000 ; 1 ; 47(2): 319-25$.

7. Gray JR, McCormick B, Cox L, et al. Primary breast irradiation in large-breasted or heavy women: değerlendirmelerimizde doz dağılımının homojen olduğu, karşı meme dışındaki kritik organ dozlarının düşük olduğu gözlemlenmiştir.

\section{SONUC}

Panduler memelerin supine pozisyonda alınması ile daha çok doz inhomojenitesi görülmekte ve bunun sonucu olarak kozmezis bozulmaktadır. Ayrıca büyük alanların 1şınlanması ile akciğer ve kalp toksisitesi artmaktadır. Prone pozisyonda sabitlenen pandüler memelerde set up farklılıkları oluşmamakta ve meme sulkusu korunabilmektedir. Hastalarımızda prone pozisyonun dezavantajı karşı meme maksimum dozunu düşürememek (ortalama meme hacmi 2274 cc olan 7 hastada tolerans dozu aşıldi) oldu. Ancak akciğer ve kalp dozları oldukça düşük bulundu (akciğer V20 ortalama \%5, Kalp mean ortalama 256 cGy). Prone pozisyon panduler meme RT 'sinde uygulanabilir bir teknik olarak değerlendirilebilir, özellikle meme sulkusu korunması, akciğer ve kalp dozlarının düşürülmesi açısından prone pozisyonu önermekteyiz.
Analysis of cosmetic outcome. Int $\mathbf{J}$ Radiat Oncol Biol Phys. 1991; 21:347-354.

8. Moody A.M , Mayles WPM, Bliss JM et al. The influence of breast size on late radiation effects and association with radiotherapy dose inhomogogeneity .Radiotherapy and Oncology 1994 ;33(2) :106-112

9. Cox JD, Stetz J, Pajak TF.Toxicity criteria of the Radiation Therapy Oncology Group (RTOG) and the European Organization for Research and Treatment of Cancer (EORTC) Int J Radiat Oncol Biol Phys. 1995;31(5):1341-6

10. Clarke D.,Martinez A., CoxRS.,Analysis of cosmetic results and complications in patients with stage I and II breast cancer treated by biopsy and irradiation. Int J Radiat Oncol Biol Phys.1983;9:1807-1813.

11. Ray GR ,FishVJ.Biopsy and definitive radiation therapy in stage I and II adenocarcinoma of breast: analysis of cosmesis and the role of electron beam supplementation. Int $\mathrm{J}$ Radiat Oncol Biol Phys.1983;9:813-818.

12. Bergom C.,Kelly T., Morrow $\mathrm{N}$ et al. Prone whloebreast irradiation using three-dimensional conformal radiotherapy in woman undergoing breast conservation for early disease yields high rates of excellent to good cosmetic outcomes in patients with large and/or pendulous breasts. Int J RadiatOncolBiol Phys.2012;83(3)

13. ChenLY, Cheng $\mathrm{CH}$, Kuo $\mathrm{SH}$ et al.Prone breast forward intensity-modulated radiotherapy for Asian women with early left breast cancer: factors for 
cardiac sparing and clinical outcomes. Journal of RadiationResearch, 2013, 54, 899-908

14. Goodman KA, Hong L, Wagman R et al. Dosimetric analysis of a simplified intensity modulation technique for prone breast radiotherapy. Int J Radiat Oncol Biol Phys. 2004 ;60(1):95-102 